$\begin{array}{cl}\begin{array}{c}\text { Revue } \\ \text { de } / \text { histoire } \\ \text { des religions }\end{array} & \text { Revue de l'histoire des religions } \\ & \begin{array}{l}1 \mid 2013 \\ \text { Varia }\end{array}\end{array}$

\title{
Fissures et paradoxes dans la théologie politique d'Élie Merlat (1634-1705)
}

Rupture and paradox in the political theology of Élie Merlat

(1634-1705)

Myriam Yardeni

\section{(Q) OpenEdition}

Journals

Édition électronique

URL : http://journals.openedition.org/rhr/8061

DOI : $10.4000 /$ rhr.806

ISSN : 2105-2573

Éditeur

Armand Colin

Édition imprimée

Date de publication : 1 mars 2013

Pagination : 67-84

ISBN : 978-2-200-92863-6

ISSN : 0035-1423

\section{Référence électronique}

Myriam Yardeni, «Fissures et paradoxes dans la théologie politique d'Élie Merlat (1634-1705) », Revue

de l'histoire des religions [En ligne], 1 | 2013, mis en ligne le 01 mars 2016, consulté le 19 avril 2019

URL : http://journals.openedition.org/rhr/8061 ; DOI : 10.4000/rhr.8061 


\section{Fissures et paradoxes dans la théologie politique d'Élie Merlat (1634-1705)}

Élie Merlat est considéré comme l'un des théoriciens les plus importants de l'absolutisme. Pourtant, son Traité du pouvoir absolu des Souverains n'est pas un livre de pensée politique ni de théorie politique. $C$ 'est un ouvrage de théologie profondément imprégné de piété et de charité chrétienne. Son but est d'œuvrer au salut de ses frères huguenots cruellement persécutés.

\section{Rupture and paradox in the political theology of Élie Merlat} (1634-1705)

Élie Merlat is one of the most important theoreticians of absolutism. Nevertheless his work Traité du pouvoir absolu des Souverains is neither a book of political thought nor of political theory. It is a book of theology deeply impregnated with Christian piety and charity. His aim is to help his cruelly persecuted Huguenot brothers not to endanger their salvation. 
La «carrière» du Traité du pouvoir absolu des Souverains ${ }^{1}$ d'Élie Merlat s'ouvre sous de bons augures, car c'est Pierre Bayle qui se charge de lui trouver un éditeur et c'est le philosophe de Rotterdam qui annonce la parution de l'ouvrage à l'occasion d'un compte rendu extrêmement élogieux paru dans ses Nouvelles de la République des Lettres lors de la livraison d'août $1685^{2}$, soit un mois avant la publication même du livre et à peine deux mois avant la révocation de l'Édit de Nantes.

Ce Traité renforce les thèses absolutistes de Bayle, bien que Merlat aille beaucoup plus loin sur ce sujet ${ }^{3}$. D' après lui, le pouvoir politique est toujours, en toutes circonstances et quelles que soient les conditions, sans bornes ni restrictions. On pourrait même qualifier son Traité des plus absolutistes parmi les écrits politiques du temps, plus absolutiste que le Léviathan de Hobbes que Merlat connaît et mentionne, et plus absolutiste déjà que la Politique tirée des propres paroles de l'Écriture Sainte de Bossuet qui paraît en $1706^{4}$. Hubert Bost le désigne à la fois comme «quintessence et caricature de la littérature huguenote anti-monarchomaque du XVII ${ }^{\mathrm{e}}$ siècle $»^{5}$. Si Merlat figure presque toujours parmi les théoriciens importants de l'absolutisme auquel les spécialistes de l'histoire de la pensée politique consacrent quelques pages ou quelques lignes, aucune monographie d'ampleur n'a été consacrée directement à la spécificité de sa pensée et de sa conception du pouvoir temporel ${ }^{6}$.

1. Élie Merlat, Traité du pouvoir absolu des Souverains. Pour servir d'instruction, de consolation et d'Apologie aux Eglises Réformées de France qui sont affligées, à Cologne, Chez Jacques Cassander, 1685.

2. Nouvelles de la République des Lettres, 1685, Article VII (p. 352-354).

3. Sur cette communion d'idées, voir Hubert Bost, «Entre droit et religion: obligation de conscience et obéissance civile», dans id., Pierre Bayle historien, critique et moraliste, Turnhout, Brepols, 2006, p. 217.

4. Voir l'Introduction de Jacques Le Brun à l'édition de ce texte, Genève, Droz, 1967.

5. Hubert Bost (en collaboration avec Didier Poton), «Élie Merlat ou la fin du monde», dans id., Ces Messieurs de la R.P.R. Histoire et écritures de huguenots, XVII ${ }^{e}$ XVIII ${ }^{e}$ siècles, Paris, Honoré Champion, 2001, p. 149-174.

6. Voir néanmoins Alfred Galland, «Les pasteurs français (Amyraut, Bochart, [Merlat] etc.) et la royauté de droit divin de l'édit d'Alais à la Révocation (16291685)», BSHPF 77 (1928), p. 14-20, 105-134, 225-241, 413-423. Nous n'avons pu consulter: Andreas Künneth, Leben und Werk des Hugenotten Pfarrer Elie Merlat (1634-1705), Orthodoxer Theologe, Staatstheoretiker und Glaubensflüchtling, Munich, 1994 (probablement s'agit-il d'une thèse non éditée). 
Pourtant, Élie Merlat est plus qu'un simple représentant caractéristique de la communauté des pasteurs exilés au Refuge. Il est un théologien original et important, un produit typique de l'Académie de Saumur, disciple d'Amyraut et de Cappel ${ }^{7}$. La première partie de sa carrière se déroule à Saintes où il est nommé pasteur en 1658. En 1676 il publie à Saumur une Réponse générale au livre de M. Arnauld intitulé Le Renversement de la morale de Jésus Christ. D'après Jacob Bayle, ce livre est à l'origine de l'animosité de Pierre Jurieu, qui lui aussi répond à Arnauld ${ }^{8}$. À ceci s'ajoutent aussi bien sûr les sympathies «monarchomaques» de Jurieu ${ }^{9}$. La réponse de Merlat à Arnauld déclenche le courroux de l'évêque de Saintes qui se plaint du pasteur. Merlat est alors emprisonné, torturé et finalement banni du royaume à perpétuité en $1680^{10}$. Refugié en Suisse, il est nommé pasteur et professeur à l'Académie de Lausanne, puis termine sa vie comme professeur à l'Académie de Berne.

\section{UNE GUVRE DE CIRCONSTANCE}

Les failles, les paradoxes et les bizarreries du Traité de Merlat s'expliquent par le fait qu'il s'agit d'une œuvre de circonstance, quoique mûrie et réfléchie de longue date. Merlat lui-même explique cette contradiction dans son Avertissement au Lecteur:

7. François Laplanche consacre à la théologie de Merlat plusieurs analyses importantes dans son livre L'Écriture, le sacré et l'histoire. Érudits et politiques protestants devant la Bible en France au XVII siècle, Amsterdam et Maarssen, APA University Press of Holland, 1986. Voir aussi Walter Grossmann, «Elie Merlat on Discernement of False Inspiration », Revue de Synthèse 111 (1990), p. 423-433 sur la révolte des Camisards.

8. Correspondance de Pierre Bayle, t. II, lettres 66-146 publiées et annotées par Élisabeth Labrousse et al., Oxford, Voltaire Foundation, 1999 p. 77, n. 13.

9. Sur la pensée politique de Jurieu: Guy Howard Dodge, The Political Theory of the Huguenots of the Dispersion, (with special reference to Pierre Jurieu), New York, 1947 (reprint 1967); Élisabeth Labrousse, «La doctrine politique des huguenots, 1630-1685» dans id., Conscience et conviction. Études sur le XVII ${ }^{e}$ siècle, Oxford, Voltaire Foundation, 1996.

10. Didier Poton, «Élie Merlat pasteur de l'Église réformée de Saintes (16581680). Une victime consentante de la justice du roi?», dans Benoît Garnot (dir.), Les victimes, des oubliées de l'histoire? Rennes, Presses Universitaires de Rennes, 2001, p. 381-390. On trouve de larges extraits du procès de Merlat sur Internet (Histoire-Passion) qui reprend le texte publié dans la Revue de l'Aunis et de la Saintonge, La Rochelle, 1866. 
Il y a près de quatre ans, que cet ouvrage fut tracé, et comme dessigné. De très importantes raisons le firent en suite abandonner à son Auteur [...] ayant résolu de ne le publier jamais. Des raisons contraires l'obligent aujourd'hui à changer de sentiment; ne pouvant souffrir qu'un danger imaginaire, expose sa Religion à la calomnie, et prive ses frères d'une instruction, et d'une consolation qu'il leur croit nécessaire. Le sort est donc jetté [...].

À ces objectifs, il faut ajouter aussi l'apologie, qui figure dans le sous-titre de son ouvrage. Les visées difficilement conciliables de Merlat - surtout par l'apologie adressée à Louis XIV -, qui cherche à démontrer au roi l'innocence de ses coreligionnaires sous la forme déguisée d'un traité de pensée politique, expliquent le caractère hybride de l'ouvrage. Merlat tente aussi de concilier plusieurs genres et modes d'écriture, qui se réduisent souvent à une egohistoire, voire à une ego-religion. Cette ego-histoire rend compte de ses propres expériences et de ses souffrances personnelles, de sa tentation du péché durant son emprisonnement et sous la torture ${ }^{11}$ et de sa conversion à la religion de l'absolutisme, car pour lui l'absolutisme n'est pas un régime politique, mais une forme de religion: la seule authentique et digne d'un vrai chrétien. À ce titre, le Traité devient aussi un grand texte de conversion. On y trouve les déchirements et les «discussions» qu'il a eues avec lui-même pour se convaincre et transformer ses propres expériences en leçons universelles pour ses coreligionnaires réformés.

Prenons quelques exemples. La gloire du martyre est d'ordinaire le plus haut point d'honneur et de bonheur auquel un homme de bien puisse aspirer. Cependant, sous sa plume, c'est un faux zèle qui entraîne la mort spirituelle et transforme le chrétien en un révolté opiniâtre et criminel $^{12}$. Pour Merlat, la seule solution réside dans la souffrance passive sans bornes, car le pouvoir absolu qui est à l'origine de ces souffrances est la volonté même de Dieu. Les révoltés ne souffrent pas «pour Jésus Christ, mais pour leurs pechez propres $\gg^{13}$.

11. «J'avois de la peine à me persuader qu'il ne fut pas permis de haïr ceux que je considerois comme ennemis de Dieu... Je ne croi $[$ sic $]$ pas faire de tort à mes Frères les plus sanctifiez, en leur attribuant des foiblesses, sinon pareilles, pour le moins semblables aux miennes...»: Traité du pouvoir absolu, op. cit., p. 8.

12. Ibid., p. 5-6.

13. Ibid., p. 13. 
En une longue suite de chapitres ${ }^{14}$ qui égrènent toutes les raisons spirituelles et temporelles démontrant que l'absolutisme sans bornes et sans restrictions est le seul régime politique juste et concevable pour un vrai chrétien, Merlat cherche à convertir ses frères persécutés à cette religion très individuelle, à la fois pour les sauver physiquement ici-bas et spirituellement dans l'au-delà. Pour concilier des buts inconciliables, le Traité se présente aussi sous la forme d'un manuel de science politique et d'un catéchisme déguisé.

La sincérité de Merlat ne fait pas de doute; il n'attend rien en échange, avouant que «sortant de France, nous avons été humainement recueillis [...] nous avons tout sujet de flatter le Gouvernement Républiquain dont nous éprouvons la douceur $»^{15}$. Pour Pierre Bayle, ce fait renforce les arguments absolutistes de Merlat: "C'est donc la forme de la persuasion qui le fait parler et qui l'oblige à fortifier ses frères dans une vérité, qu'il regarde comme de la dernière importance $»^{16}$. Euvre de circonstance, le Traité de Merlat vise néanmoins à aboutir à la compréhension universelle conjointe de l'histoire et de la religion.

\section{Temps et espace dans la conception de l'Histoire de Merlat}

Pour Merlat, l'histoire humaine (passée ou immédiate) n'est que la continuation de l'histoire sainte. Le temps et l'espace dans lesquels se déroule cette histoire sont chez lui des entités irréelles et imaginaires, voire intemporelles. L'histoire d'ici-bas n'est qu'une préparation aux multiples facettes de la vie éternelle, à tout le moins pour les élus. Selon lui, la réalité se dégage de la portée théologique et religieuse de l'histoire.

Cette histoire se divise en trois périodes: celle de la Création et du péché originel, celle de la raison (c'est-à-dire de l'Ancien

14. Voir la succession des chapitres:

Chapitre premier. Dessein de cet Ouvrage. - Chapitre II. Etat de la question touchant le pouvoir absolu du Souverain. - Chapitre III. Première preuve tirée des maximes générales de l'Ecriture. - Chapitre IV. Seconde preuve tirée des maximes particulières de l'Evangile. - Chapitre V. Troisième preuve tirée du fondement du Droit. - Chapitre VI. Quatrième et dernière preuve, tirée de l'origine des Puissances. - Chapitre VII. Où il est satisfait aux scrupules de la conscience.

15. Traité du pouvoir absolu, op. cit., p. 22.

16. Nouvelles de la République des Lettres, op. cit., p. 354. 
Testament) et celle de la Grâce (ou du Nouveau Testament ${ }^{17}$. La distinction s'opère d'après le degré de la volonté libre que Dieu accorde à l'homme.

Selon la tradition calvinienne, le péché originel fournit à Merlat l'explication de la dynamique et de «l'autonomie» de l'histoire, dans les limites que Dieu accorde à l'homme:

les événemens sont des effets de la Providence de Dieu, et des preuves de sa volonté secrette, et non pas des règles de la conduite des hommes. L'homme pèche donc en violant la parole de Dieu, quoy que son péché n'ai pû arriver sans l'ordre et sans la Providence secrette de Dieu, et Dieu ne se contredit point ${ }^{18}$.

Malgré cette constatation, après la Création, l'homme est toutefois libre de prendre ses propres décisions. Il y a ici une certaine confusion dans les explications de Merlat, car sur le plan historique, il parle des peuples et d'un dynamisme collectif, voire même d'une élection collective. Or l'idée de l'élection collective après la chute des Juifs est vigoureusement rejetée par tous les théologiens calvinistes, y compris par Moïse Amyraut, le maître de Merlat $^{19}$.

Selon Merlat: «tous les hommes sont égaux par la nature: ils naissent, et meurent d'une même manière [...] il n'y doit avoir entre eux, ni dépendance, ni domination que celle qui fait la différence des pères et des enfans, ou la diversité des dons naturels $»^{20}$. Le péché a bouleversé l'ordre de la Création: «ayant détruit l'image de Dieu dans l'homme, introduit la confusion au lieu de l'ordre, et assujettit la raison aux passions [...] les hommes n'ont donné d'autres bornes à leurs désirs et à leurs entreprises, que celle de leur impuissance $»^{21}$. Dieu a sauvé l'humanité en mettant fin à l'anarchie, bridant cet instinct d'autodestruction en lui apportant le pouvoir absolu, sans

17. Cette périodisation est à contre-courant de ce qui se passe dans la pensée historique et théologique huguenote de l'époque: Nicolas Piqué, De la tradition à l'histoire. Éléments pour une généalogie du concept de l'histoire à partir des controverses religieuses en France (1669-1709), Paris, Honoré Champion, 2009.

18. Traité du pouvoir absolu, op. cit., p. 290.

19. «Le salut n'est ni pour les Italiens, ni pour les François, en tant qu' ils sont ou François ou Italiens, mais seulement pour les vrais Chrestiens. Et les vrais Chrestiens sont ceux là les seuls qui ont à cœur la religion chrestienne avec connoissance»: Moyse Amyraut, Discours de l'élévation de la foy et de l'abaissement de la raison en la créance des mystères de la religion, Saumur, 1640, p. 76.

20. Traité du pouvoir absolu, op. cit., p. 23-24.

21. Ibid., p. 24-25. 
conditions et sans bornes. Et néanmoins, certains peuples vivent sous des régimes plus libres et plus doux et sont plus heureux que d'autres: le pouvoir absolu s'est établi par degrés. La diversité des gouvernements vient de la diversité des peuples: «La douceur de l'Aristocratie, ou la liberté de la Démocratie, n'ont lieu que dans les Etats, où l'ambition particulière est moindre, et où l'égalité est plus aisément observée $»^{22}$.

Cependant, cette «élection» collective - ou bien cette volonté libre collective - n'a rien à voir avec la chronologie historique de Merlat, qui est double et qui va, pour ainsi dire, dans deux sens. Il y a ainsi une dégradation continuelle qui passe de la Création par l'âge de la raison à l'âge de la Grâce. C'est l'âge de la Grâce qui, en fin de compte, symbolise et signifie le comble du "progrès» de l'histoire humaine, car c'est lui qui approche l'élu de la vie éternelle: "La félicité qui vient de Christ, élève les Disciples de la grâce aux biens du monde à venir [...]. Voilà pourquoy encore les Chrêtiens sont considérez comme Pélerins et Etrangers dans ce monde $»^{23}$.

Pour le bon chrétien et le bon calviniste qu'est Merlat, l'histoire humaine n'est que la phase préliminaire de la vie éternelle des élus. Elle n'a de sens et d'autonomie que si elle fait partie de l'histoire sainte, en la prolongeant. C'est cette conception de l'Histoire qui sert d'écrin à la théologie politique de Merlat.

\section{RELIGION ET THÉOLOGIE POLITIQUE}

La science politique et la philosophie politique se réduisent chez Élie Merlat à une théologie politique. Cette théologie puise ses racines dans sa religion, ses croyances et sa foi. Son argumentation est d'ordre théologique. Elle se fonde sur le principe selon lequel l'absolutisme (ou le pouvoir absolu comme ill' appelle) est infiniment

22. Ibid., p. 29. La France appartient à ces peuples d'une «humeur ambitieuse», écrit Merlat, «une Autorité limitée ne les sçauroit gouverner; et si leurs Souverains étoient moins absolus, leurs Etats seroient incontinent remplis de séditions » (ibid., p. 29-30).

23. Ibid., p. 94. Sur les changements survenus dans ce domaine: Hubert Bost, ""Étrangers et voyageurs", mais patriotes: Exil et refuge dans le discours huguenot», dans Ces messieurs de la R.P.R., op. cit., p. 281-302. 
plus désirable pour la société civile que l'anarchie dans laquelle plongent inévitablement tous les régimes où les sujets peuvent exprimer leurs opinions et, pire encore, critiquer le souverain: «le droit d'examen une fois concédé aux peuples sur leurs Princes dans la Société civile, détruit la Puissance et fait l'Anarchie $»^{24}$. C'est le pouvoir absolu qui sauve la société civile de la décomposition et du désordre le plus total. Bien plus, c'est un don de Dieu, une grâce accordée aux hommes après le péché originel, qui leur permet de mener leur vie dans des conditions supportables, jusqu'à ce que les élus accèdent à la vie éternelle.

Pour Merlat, tout pouvoir politique doit être absolu, peu importe le type de régime - monarchie absolue, monarchie héréditaire ou monarchie élective - qui l'exerce. Les sujets doivent se comporter à l'égard de leur souverain comme s'il s'agissait du pouvoir le plus absolu, même s'il est clément. Cette relative mansuétude est fonction directe de la culpabilité des peuples qui composent les différents types de monarchies. Bien sûr, les républiques, tenues pour foyers d'anarchie, n'appartiennent pas à la catégorie des régimes ordonnés, même si elles sont imposées, comme tous les régimes politiques, par Dieu.

Les sujets doivent s'adapter à chaque type de régime, sans vouloir y apporter de changements car, selon Élie Merlat, Dieu est pour l'immobilisme de l'homme, qui est le signe de son obéissance et de sa foi. Dans ce système de pensée, le changement en Histoire - et celui des régimes politiques - ne peut venir que de Dieu. Dans le Traité, ceci s'applique aussi bien aux régimes les moins pesants qu' aux régimes les plus injustes ${ }^{25}$. La Providence est toujours bonne, même si ses instruments sont méchants ${ }^{26}$.

Parmi ces régimes injustes, Merlat compte les pays conquis et les grands empires formés par les guerres qui justifient l'esclavage ${ }^{27}$. On ne peut se rebeller contre un conquérant du fait qu'il lui a été promis, au moment de la conquête, une fidélité

24. Traité du pouvoir absolu, op. cit., p. 167.

25. «[Les peuples] bienheureux, qui n'ont pas besoin d'un joug si pesant, que Dieu, qui les exempte, fait voir par là que leur corruption est encore moindre que celle des Peuples qui le sentent; et qu'il les convie aussi, à même temps, à ne pas abuser de leur liberté, de peur que sa sage et juste Providence ne les en prive, et ne les réduise au pied des autres »: Traité du pouvoir absolu, op. cit., p. 321).

26. Ibid., p. 161.

27. Ibid., p. 201. 
inviolable ${ }^{28}$. Les puissances usurpées par ruse entrent dans cette même catégorie ${ }^{29}$.

L'argumentation religieuse de Merlat s'adresse à deux «publics » diamétralement opposés: d'une part, à ses coreligionnaires restés en France, qu'il estime en danger de mort physique et spirituelle, et d'autre part, à Louis XIV qu'il cherche à persuader du fait qu' aucune rébellion à sa politique anti-calviniste et à ses persécutions ne peut entraîner une révolte huguenote qui serait totalement étrangère aux enseignements de la religion réformée.

Dans cette perspective, Merlat traite de deux thèmes majeurs: de la révolte armée des huguenots à l'époque des guerres de Religion et des thèses monarchomaques. De manière générale, ces guerres qu'on appelle guerres de Religion furent simplement des guerres politiques. En Allemagne, comme en Suède et ailleurs, les souverains utilisaient le prétexte religieux pour envahir les territoires de leurs voisins: «une partie des guerres de France tombent aussi sous ce genre sçavoir celle de Henry Quatrième contre la Ligue, et celle de 1562, entreprise par le Prince de Condé, contre la Maison de Lorraine, à la prière de Catherine de Médicis, Reine de France alors Régente $»^{30}$. Merlat concède, que même de son temps, des guerres religieuses justes éclatent, à l'instar de celle des Vaudois, mais que c'est là l'exception. Bien plus. Ceux qui pensaient mourir pour le Christ et leur croyance à l'époque des guerres de Religion n'étaient que de simples pécheurs ${ }^{31}$.

La campagne qu'il mène contre les anciens et les nouveaux monarchomaques est plus complexe. On sait que les grands monarchomaques $\mathrm{du} \mathrm{XVI}^{\mathrm{e}}$ siècle tiraient leurs justifications de l'Ancien Testament et de la révolte des Maccabées ${ }^{32}$. Merlat ne peut évidemment pas condamner cette révolte: «La rebellion fut legitime sous l'Ancienne Alliance: et les Maccabées furent louables d'avoir

28. Ibid., p. 202-204.

29. Ibid., p. 205-206.

30. Ibid., p. 255.

31. Ibid., p. 306-307.

32. La littérature sur le sujet est immense. Pour une synthèse, voir la thèse récente de Paul-Alexis Mellet, Les traités monarchomaques: Confusions des temps, résistance armée et monarchie parfaite (1560-1600), Genève, Droz, 2007, qui discute de l'ensemble des thèses et dont la bibliographie exhaustive englobe toutes les références sur le sujet. 
exposé leurs biens et leurs vies pour cela $»^{33}$. Mais il s'agissait d'autres temps et d'autres circonstances. Et l'auteur de poursuivre:

chacun sçait que la dernière destruction de Jerusalem a eu pour cause civile, la rébellion des Juifs contre les Romains; quoy que cette rebellion eut été produite par les traitemens les plus violens et les plus tyranniques dont on ait jamais ouï parler ${ }^{34}$.

Pour Merlat, les autres temps et les autres circonstances se résument à la supériorité de l'Évangile, de l'époque de la Grâce, sur l'Ancien Testament de l'époque de la raison. Ici, Merlat renoue avec la tradition séculaire de l'Église catholique. Bien plus encore, c'est ici un fossé qui le sépare de Calvin.

\section{UNE THÉOLOGIE POLITIQUe À L'USAGE DES «MONDAINS 》}

Les chapitres que Merlat rédige à l'usage des «mondains $»^{35}$ masquent efficacement le caractère foncièrement religieux du Traité en le transformant en manuel de science politique. En même temps, ce sont aussi les chapitres les plus originaux de son ouvrage.

Les mondains que Merlat veut convaincre par des preuves tirées du droit universel et de l'histoire humaine sont ceux qui ne se contentent pas d'arguments basés uniquement sur les Écritures saintes. Il cherche à leur démontrer que l'on peut aussi fonder la légitimité du pouvoir absolu sur son universalité et son utilité. Or, depuis La crise de la conscience européenne de Paul Hazard ${ }^{36}$, on sait qu'une large majorité d'adeptes et de convaincus n'attestent pas la justesse et la vérité d'une thèse, d'une théorie ou d'une doctrine. La raison chasse la majorité chez tous ces sceptiques et mondains, y compris Bayle.

À cause de son immobilisme et anachronisme, Merlat ne comprend pas comme Bayle que depuis l'Humanisme et la Renaissance,

33. Traité du pouvoir absolu, op. cit., p. 248.

34. Ibid., p. 249.

35. Voici comment La Bruyère définit les mondains: «J'appelle mondains, terrestres ou grossiers, ceux dont l'esprit et le cœur sont attaches a une petite portion de ce monde qu'ils habitent, qui est la terre: qui n'estiment rien, qui n'aiment rien au-delà, gens aussi limites que ce qu'ils appellent leurs possessions ou leur domaine»: Caractères, chap. XVI, 3). Cf. Didier Boisson, Consciences en liberté? Itinéraires d'ecclésiastiques convertis au protestantisme, 1631-1760, Paris, Honoré Champion, 2009, p. 7.

36. Paul Hazard, La crise de la conscience européenne, 3 vol., Paris, Boivin et Cie, 1935. 
l'individualisme, l'esprit critique et la raison commencent à renverser l'ordre traditionnel des choses. Que les «vérités» inébranlables établies par des autorités collectives comme les «Anciens» ou l'Église catholique peuvent s'écrouler sous le faix de nouveaux raisonnements et déductions individuels. Il est vrai que Bayle et Merlat diagnostiquent sans faille le potentiel subversif de la raison et de l'esprit critique et les dangers qu'ils représentent pour les régimes politiques. Ce qui est l'une des causes pour laquelle ils privilégient l'absolutisme, la meilleure sauvegarde des horreurs de l'anarchie.

À l'encontre de Bayle, Merlat ne veut pas et ne peut pas accepter le règne de la raison. Même lorsqu'il propose des explications originales et «convaincantes» sur les origines et l'universalité du pouvoir absolu, il est évident que pour lui c'est Dieu qui est la cause première de tout ce qui se passe dans le monde qu'il a créé.

Les explications de Merlat sont d'ordre anthropologique, psychologique, philosophique et éthique. Ainsi, la force des lois n'est pas dans la justice mais dans l'autorité ${ }^{37}$. Il démontre, notamment dans le cas de David, que la raison d'État oblige les princes à commettre des injustices ${ }^{38}$, mais que les renverser revient à renverser la société civile ${ }^{39}$. Les actes d'injustice des princes, commis au nom de la raison d'État ont leur origine dans l'autorité qui caractérise leurs fonctions, car la souveraineté du monarque le place «dans un degré d'éminence qui ôte toute proportion entre lui et ses sujets $»^{40}$. C'est l'éminence qui entraîne l'adoration civile et rend possible l'établissement et la sauvegarde du pouvoir illimité. Merlat dresse une comparaison extrêmement pertinente entre l'adoration de Dieu et celle du roi, en affirmant la légitimité du pouvoir absolu par le biais de la vénération. Pour lui, l'adoration religieuse et l'adulation civile fonctionnent de la même manière.

Il faut que l'une et l'autre renferment une espèce d' anéantissement, et de sujettion sans réserve, de la chose qui adore, à l'égard de celle qui est adorée; et l'une et l'autre supposent dans la chose adorée un Pouvoir de détruire absolument celle qui l'adore; tellement que c'est, dans une soumission volontaire à cette puissance de détruire, que consiste proprement l'acte d'adoration. Comme donc l'on adore Dieu religieusement en lui soumettant l'âme, et la conscience, sur

37. Traité du pouvoir absolu, op. cit., p. 159.

38. Ibid., p. 159.

39. Ibid., p. 161.

40. Ibid., p. 149. 
lesquels il règne; aussi en adorant les Rois, on reconnoît qu'ils ont une puissance absoluë sur les corps, et sur la vie presente, ce qui réduit leur adoration au genre politique ou civil; et prouve par elle, la même éminence dans les Rois quant aux choses temporelles, que celle qui est en Dieu quant aux choses de la conscience, que nous considérons comme éternelles. ${ }^{41}$

Le parallélisme entre ces deux sortes d'adoration confère à l'analyse une dimension anthropologique et ouvre la piste à d'autres interprétations de l'acte du pouvoir qui servent les buts que Merlat veut atteindre: servir le protestantisme et sauver les croyants et les églises persécutées, même sur le plan conceptuel.

\section{Le Calvinisme de Merlat}

Personne ne mettra en doute l'authenticité du calvinisme de Merlat, ni sa fidélité aux principes de la Réformation dont témoignent irréfutablement sa vie, son bannissement de sa patrie et jusqu'à son Traité du pouvoir absolu des souverains. Mais est-ce pour autant un disciple inconditionnel de Calvin?

On sait que le calvinisme, comme tout phénomène historique, évolue selon les circonstances, les lieux et les temps ${ }^{42}$. Le calvinisme de Merlat est aussi un calvinisme de circonstances. De là ses ambigüités. Il manifeste à diverses occasions une proximité spirituelle et idéologique à toute épreuve avec Calvin, notamment quant à l'omniprésence du péché originel dans la vie des individus, voire dans sa considération du péché comme cause unique et comme explication du monde et de l'histoire humaine. Pourtant, même s'il parle dans le Traité des modalités et des formes d'élection ${ }^{43}$, cette question est moins centrale chez lui que chez Calvin.

Chez Merlat, foi et obéissance se confondent et l'on pourrait même dire que sur ce point il est plus calviniste que Calvin lui-même ${ }^{44}$.

41. Ibid., p. 156-157.

42. Menna Prestwich (ed.), International Calvinism (1541-1715), Cambridge, Clarendon Press, 1985; W. Fred Graham (ed.), Later Calvinism. International Perspectives, Kirksville, Sixteenth Century Essays and Studies, vol. XXII, 1994; Richard A. Muller, After Calvin. Studies in the Development of a Theological Tradition, Oxford, O.U.P., 2003.

43. Traité du pouvoir absolu, op. cit., p. 110.

44. H. Bost, «Élie Merlat et la fin du monde», art. cité, montre que chez Calvin l'Église exerce une fonction de vigilance critique et d'exhortation à l'égard du 
En regard des solutions présentées au persécuté, il suit de près les propositions du Réformateur de Genève:

$\mathrm{Si}$ ceux que ce Pouvoir oppresse sont réduits aux dernières extremitez, comme est celle de ne point vivre, ou de courir risque de succomber au péril de leur conscience, ils peuvent alors non pas se rebeller, en violant la puissance, mais s'enfuir, s'il leur est possible en évitant le danger de leur salut, et le mal de leur mort temporelle ${ }^{45}$.

Chez Calvin, le martyre revêt une valeur suprême liée aux premiers chrétiens persécutés ${ }^{46}$. Pour Merlat,

il n'y a rien de plus déplorable que la condition d'un homme, que le faux zèle anime à la mort de qui la bonne intention dégénère en pure hypocrisie, et qui fait d'une rebellion opiniâtre et criminelle, une action de piété héroïque ${ }^{47}$.

On connaît la critique que Calvin exerce à l'égard des nicodémites ${ }^{48}$. Chez Merlat, on peut difficilement distinguer entre obéissance et nicodémisme. Sur le problème de la tolérance, Merlat est curieusement plus libéral que Calvin, et loue ses avantages pour la société civile ${ }^{49}$. Par contre, il est contre toute tentative et forme de persuasion en matière de religion au nom même de l'immobilisme qui signifie pour lui l'obéissance et la soumission parfaite à la volonté divine ${ }^{50}$. Mais c'est la position à l'égard des deux Testaments qui sépare le plus profondément Merlat de Calvin, au-delà de la théologie des alliances de Moïse Amyraut et de l'école de Saumur ${ }^{51}$.

Pour Calvin le «judaïsant» les deux Testaments ont la même valeur de témoignage permettant d'authentifier la foi des élus, le

pouvoir, tandis que Merlat restreint le spirituel à la sphère individuelle et tend à donner au souverain une vision messianique. Se rebeller contre lui, c'est se rebeller contre Dieu (p. 163-164). Voir aussi Max Engammare, «Calvin monarchomaque, du soupçon à l'argument», Archiv für Reformationsgeschichte 89 (1998), p. 207-226.

45. Traité du pouvoir absolu, op. cit., p. 289.

46. Pierre Janton, «La non-violence et le martyre chez Calvin», BSHPF 157 (2011), p. 295-308.

47. Traité du pouvoir absolu, op. cit., p. 6.

48. Carlos M.N. Eire, «Calvin and Nicodemism: A Reappraisal», Sixteenth Century Journal 10 (1979), p. 45-69.

49. Traité du pouvoir absolu, op. cit., p. 329.

50. «Ainsi, il est indubitable que quiconque est persuadé de la vérité d'une religion, doit y demeurer immuable» (ibid., p. 34) «[...] Et ceci concerne non seulement l'autorité, et la force, mais aussi la persuasion». Sur ce point, il est étonnamment proche de la conscience errante de Bayle.

51. F. Laplanche, L'Écriture, le sacré et l'histoire, op. cit., p. 440-445, 650 sq. 
Réformateur plaçant les deux livres saints sur un pied d'égalité 52. On connaît aussi l'identification profonde de Calvin aux Juifs et au judaïsme de l'Ancien Testament. Chez Merlat, rien de semblable. La supériorité de l'Évangile est la pierre de touche de sa religion et de sa conception de l'histoire. Pour lui, la révélation s'est faite par degrés ${ }^{53}$ et pour aboutir à Jésus-Christ, il aura fallu le péché et la Rédemption ${ }^{54}$. De là sa périodisation, qui, partant du péché, passe par l'âge de la raison pour aboutir à Jésus à l'âge de la Grâce. Par conséquent, les preuves tirées de l'Évangile sont pour lui plus convaincantes que celles tirées de l'Ancien Testament.

Ajoutant la grâce à la Nature, pour empêcher la récidive, au lieu des lumières de la simple raison pour la connoissance, il [Dieu] a donné la révélation de la Foy; au lieu des maximes de la justice pour l'action, il a donné les préceptes de la charité; et au lieu des vues de la vie terrienne et naturelle pour le bonheur, il a donné les promesses de la gloire du $\mathrm{Ciel}^{55}$.

Il est clair que dans cette hiérarchie, l'Ancien Testament est bien en dessous du Nouveau. Ceci devient encore plus évident lorsqu'il traite du problème de la rébellion dans l'Ancien Testament. Les Juifs ont pu se rebeller à cause de la mission extraordinaire des prophètes, mais depuis la venue de Jésus-Christ - et c'est là une thèse protestante présente dans tous les textes, toutes tendances protestantes confondues: «cette mission extraordinaire n'a plus lieu; Les Prophètes ont cessé; Dieu nous a réduits à la révélation de sa parole comprise dans l'Écriture ${ }^{56}$. De même, les Juifs ont pu se rebeller car le judaïsme fut établi pour un certain lieu. Le judaïsme fut une religion locale et charnelle, tandis que le christianisme est une religion spirituelle et universelle.

Les juifs ne pouvoient laisser ruiner l'exterieur de leur Religion, sans blesser leur conscience. Au lieu qu'au contraire en cela qu'aujourd'hui le christianisme est tout spirituel, et ne dépend nullement quant à son essence, ni des temps ni des lieux [...] et par conséquent les chrétiens ne peuvent en aucune manière se rebeller

52. Myriam Yardeni, Huguenots et Juifs, Paris, Honoré Champion, 2008, p. 31-32.

53. Traité du pouvoir absolu, op. cit., p. 186.

54. Ibid., loc. cit., p. 186.

55. Ibid., p. 93.

56. « Sous le judaïsme, Dieu avoit attaché la Religion à un certain lieu, l'avoit rempli de cérémonies charnelles et extérieures, et avoit comme consacré le Païs de Canaan au bonheur du Peuple qu'il avoit choisi» : ibid., p. 250. 
contre leurs princes pour cause de Religion, non plus que pour aucune autre cause..$^{57}$

Plus encore, on pourrait dire que les Juifs sont devenus le peuple élu par hasard: «Le judaïsme fut établi pour la punition temporelle de l'Égypte ${ }^{58}$ sans oublier que ce fut aussi l'âge du péché, période durant laquelle l'homme disposait encore d'une volonté relativement libre. Et Merlat de conclure que Dieu procède tout autrement dans le judaïsme que dans le christianisme. Cette véritable rupture avec Calvin sur le rôle des deux Testaments dans le christianisme ne provient pas chez Élie Merlat d'un antisémitisme viscéral. Elle est le fruit des circonstances, d'un besoin pressant de se démarquer aussi fortement que possible des monarchomaques passés et présents ${ }^{59}$ qui s'inspiraient de l'exemple des Macchabées et de leur révolte au nom de la religion. Une rupture qui souligne une fois de plus le caractère essentiellement religieux du Traité.

\section{Pour un Changement de Perspective}

C'est le compte rendu de Pierre Bayle qui classe définitivement le Traité de Merlat dans la catégorie des écrits de théorie politique ${ }^{60}$. Il est vrai que le titre même de l'ouvrage met en évidence la tonalité politique de l'opuscule et que seuls les sous-titres rappellent les préoccupations religieuses de son auteur. Or, on a vu qu'il s'agit d'une œuvre dont chaque page exhale le caractère essentiellement religieux, ainsi qu'en témoignent la supériorité accordée aux preuves bibliques sur les preuves «mondaines», l'ego-histoire de Merlat en tant que pêcheur, l'urgence d'une «conversion» collective des réformés de France à cette religion de l'absolutisme préconisée par l'auteur comme la seule qui puisse les réconforter, les affermir dans leurs souffrances, et ne pas compromettre la grâce divine qui, peutêtre, les attend.

57. Ibid. p. 250.

58. Ibid. loc. cit.

59. On pense notamment à la Révolution anglaise et à Cromwell, à la discussion et aux controverses qu'ils ont suscité dans le camp huguenot. Élisabeth Labrousse, «La doctrine des huguenots: 1630-1685», Études théologiques et religieuses 47 (1972), p. 421-429.

60. Cf. supra n. 2. 
La passivité totale, voire le nicodémisme que prêche Merlat, est en contradiction flagrante avec l'esprit d'activisme calviniste «weberien». Cependant, la rédaction et la publication même de ce livre constituent paradoxalement une forme d'activisme.

Cette mise en évidence de l'aspect politique de la religion montre en fin de compte que Merlat - et peut-être avec lui une poignée de pasteurs - réalise déjà que le problème des persécutions des réformés n'est pas un problème religieux mais un problème politique, un problème de raison d'État. C'est là une considération qui est en contradiction avec ce que soutiennent les chefs spirituels du protestantisme français avant la révocation de l'édit de Nantes ${ }^{61}$. Ce n'est pas seulement le clergé catholique qui incite à l'intolérance et aux persécutions. C'est Louis XIV lui-même qu'il faut convaincre de l'innocence de ses sujets de la R.P.R. pour ses propres intérêts et pour le bien de l'État. Merlat cherche à démontrer au roi que par leur passivité et leur totale soumission à sa volonté, les protestants accomplissent un commandement divin, inébranlable et indiscutable. À ce titre, ils sont infiniment moins «dangereux» que les représentants activistes de Rome: "Il est impossible que des raisons de conscience obligent un Prince à traiter ses sujets; comme le Clergé Romain veut que le Roy de France traite les Eglises Réformées de son Royaume $»^{62}$. C'est la tolérance des deux religions qui assure la paix de l'État:

les efforts faits pour abolir l'une des Religions a mis l'État en danger, $[\ldots]$ sa fermeté est procédée de la tolérance des deux, et de la liberté de conscience ${ }^{63}$.

C'est Rome qui, malgré son affaiblissement, constitue le véritable danger pour l'État ${ }^{64}$. En revanche, le roi peut tirer une assurance absolue en la pérennité de son État avec la religion réformée. L'hybridité même du Traité avec ses failles et ses paradoxes illustrent l'ampleur de la tâche entreprise par Merlat.

61. Lettres au Clergé de France. Envoyées à ce même clergé l'an 1685, pendant qu'il était assemblé à Paris pour délibérer sur les moyens de détruire entièrement les Eglises Réformées, Genève, Émile Béroud libraire, 1853.

62. Traité du pouvoir absolu, op. cit., p. 325.

63. Ibid., p. 328-329.

64. Ibid., p. 332-333. 
Hubert Bost a remarqué à juste titre que la théorie politique de Merlat constituait déjà en son temps un pur anachronisme. D'après Merlat, le régime politique du royaume de France est une théocratie, car Dieu dirige tout et tout se passe selon sa volonté impénétrable. Mais c'est une théocratie punitive et oppressante avec pour seul espoir d'apaisement l'au-delà. Contrairement à Pierre Jurieu, pour qui le martyre apparaît dans ses Lettres pastorales comme un signe de grâce divine et, partant, comme une consolation de Dieu, Merlat considère que dans cette théocratie terrible, habitée par des nicodémites, même le martyre n'apporte pas le moindre espoir.

La théocratie de Merlat n'a rien à voir avec le monde moderne et changeant dans lequel il vit et dans lequel ses coreligionnaires sont si brutalement opprimés et persécutés. Par son extrémisme caricatural, son livre sert davantage la laïcisation que la religion.

Le Traité n'en reste pas moins digne d'intérêt. Il tient lieu de témoignage hors pair du désespoir dans lequel la politique de persécution de Louis XIV a plongé les protestants de son royaume. Ce document profondément humain et bouleversant est aussi un livre bien écrit, avec des formules et des définitions qui ont une valeur d'aphorisme et s'incrustent dans la mémoire et le subconscient. À ce titre, le Traité peut être considéré comme l'un des grands textes de la Révocation, qui mérite d'être étudié afin de mieux appréhender le vécu religieux de l'époque ${ }^{65}$.

myardeni@research.haifa.ac.il

65. Je remercie Chrystel Bernat (École pratique des Hautes Études) pour ses remarques précieuses lors de la mise au point de cet article. 